\title{
Lattice-Reduction-Aided Equalization for V2V Communication Channel
}

\author{
Samarendra Nath Sur ${ }^{\mathrm{a}^{*}}$, Rabindranath Bera ${ }^{\mathrm{a}}$, Bansibadan Maji ${ }^{\mathrm{b}}$ \\ ${ }^{a}$ Electronics and Communication Engineering Department, Sikkim Manipal Institute of Technology, Majitar, \\ Rangpo, East Sikkim-737136, India \\ ${ }^{b}$ Electronics and Communication Engineering Department, National Institute of Technology, Durgapur, West \\ Bengal, India
}

Received: 31 August 2017; Accepted: 10 November 2017; Published: 08 March 2018

\begin{abstract}
World is moving towards the implementation of massive MIMO based communication system and that forces the researchers to design low complexity receiver architecture. MIMO system performance in Vehicular ad hoc networks (VANETs) is a popular research topic. And to support Vehicle-to-vehicle (V2V) communication in high speed mobility condition, it required to have reliable and secure of com-munication. This paper deals with the performance evaluation of the low complex LLR-MMSE receiver in terms of the channel capacity and bit error rate improvement. In this paper we have considered V2V Spatial Channel Model (SCM) and Nakagami-m chaneel model for the performance evaluation. The performance has been evaluated based on the mathematical calculation and simulated results. And also performance comparison between the conventional linear MIMO receivers with the lattics reduction aided MIMO receivers have been presented.
\end{abstract}

Index Terms: MIMO, Capacity, ZF, MMSE, LLR.

(C) 2018 Published by MECS Publisher. Selection and/or peer review under responsibility of the Research Association of Modern Education and Computer Science

\section{Introduction}

From the point of view of road safety, driver protection, on the way communication etc., vehicle-to-vehicle (V2V) communication researches have gain significant importance in research world. And also today's advancement in communication technologies are moving towards the exchange of high data rate multimedia information. The growing demand of super-fast communication system leads to the development of massive

* Corresponding author.

E-mail address: samar.sur@gmail.com 
MIMO system.

The main bottleneck of this system is its complexity. And researchers have point out the importance of designing the low complex receiver system. As we know that sub-optimal receiver like linear receiver suffers from the loss of mutual information [1-3], therefore in case of a large scale MIMO system, complex Lenstra, Lenstra and Lovasz (CLLL) algorithm [4] is beneficial. And system analysis with CLLL aided receivers is become utmost important [5-9]. As in [1], capacity of MIMO using LLR-ZF receiver has been analysed. In this paper, authors have presented the improvement in MIMO channel capacity due to CLLL aided MMSE receiver (LLR-MMSE). The improvement in the channel capacity is depends on the effective utilization of the orthogonalizing of the channel matrix. LLR algorithm improves the orthogonality deficiency of the channel matrix in comparison to linear receivers [10-11]. And this helps to improve the system performance significantly over the conventional linear receivers [12-14]but with reduction in complexity w.r. to optimal receivers.

As it is already proven that MMSE perform better than the ZF receiver, here in this paper we have considered MMSE as the basic functional equalizer technique. Here, in this paper, first we have calculated the channel capacity improvement factor for LLR-MMSE receiver over the MMSE receiver. Then we have presented the performance of the LLR-MMSE receiver through the simulation. This paper also present the performance of the system with the variation in Nakagami fading parameter $\mathrm{m}$ and correlation coefficient and also in SCM channel.

The remaining paper is presented as follows. Section II mathematical formulation for capacity of LR-aided MMSE receiver for the MIMO system. While Section III represents the comparative results to evaluate the performance. Section IV provides the conclusion remarks.

\section{System Description}

\subsection{Spatial Channel Model:}

The SCM [3rd Generation Partnership Project (3GPP), 2003; J. Salo et al, 2005] is a detailed model for simulating different fading environments. It considers a large number of cluster of scatters and each cluster corresponds to a different paths. Model consist of $\mathrm{N}_{\mathrm{t}}$ element transmitter antennas and $\mathrm{N}_{\mathrm{r}}$ receiver antennas and it generate $\mathrm{N}_{\mathrm{r}} \times \mathrm{N}_{\mathrm{t}}$ matrix of complex amplitudes channel. SCM channel model takes care of some important parameter like angle of arrival (AOA), angle of departure, delay spread, Doppler spread, antenna height, path loss fading characteristic, power delay profile etc. Each those paths are characterized by its own spatial channel parameters as mentioned above. And these path are assumed to be independent. Based on the channel characteristic, there are several environments such as suburban macrocell, urban macrocell, urban microcell.

\subsection{Capacity of LR-aided MMSE receiver:}

Let us consider a MIMO system with $N_{t}$ transmitter and $N_{r}$ receiver antennas. The received signal for a MIMO system can be represented by

$$
\mathrm{y}=\mathrm{Hx}+\mathrm{w}
$$

Where $\mathrm{H}$ is the $N_{r} \times N_{t}$ complex wireless channel matrix, y represent the received signal for the given transmitted signal $\mathrm{x}$. w represent the zero mean white Gaussian noise vector having size $N_{r} \times 1$. From the simplicity point of view, here we have considered that channel sate information (CSI) is available at the receiver side but it is absent at the transmitter section.

Main aim of this paper is to analyse the capacity improvement due to LR aided MMSE receiver. Here authors have used complex LR technique. Main goal of LR aided system is convert the ill conditioned channel matrix $\mathrm{H}$ into a well-conditioned $\widetilde{\mathrm{H}}$ by exploiting orthogonalization. LR algorithm produces the reduced 
channel matrix $\widetilde{\mathrm{H}}=\mathrm{HT}$, where $\mathrm{T}$ is unimodular matrix. Therefore, in case of a LR aided MIMO system, the overall system can be represented as,

$$
y=H T\left(T^{-1} x\right)+w
$$

The channel capacity for a conventional MMSE [15] system can be represented as,

$$
C_{M M S E}=\log _{2}\left[\frac{1}{\left(I_{N_{r}}+S N R \cdot H^{H} H\right)^{-1}}\right]
$$

The channel capacity of a LR aided MMSE receiver can be represented as

$$
C_{M M S E}^{L R}=\log _{2}\left[\frac{1}{\left(I_{N_{r}}+S N R \cdot \widetilde{H}^{H} \widetilde{H}\right)^{-1}}\right]
$$

At high SNR condition, the factor $\left(I_{N_{r}}+S N R \cdot H^{H} H\right)^{-1}$ can be further expressed as

$$
\begin{aligned}
& \left(\mathrm{I}_{\mathrm{N}_{\mathrm{r}}}+\mathrm{SNR} \cdot \mathrm{H}^{\mathrm{H}} \mathrm{H}\right)^{-1}=\left(\mathrm{SNR} \cdot \mathrm{H}^{\mathrm{H}} \mathrm{H}\right)^{-1}-\mathrm{I}_{\mathrm{N}_{\mathrm{r}}}\left(\mathrm{SNR} \cdot \mathrm{H}^{\mathrm{H}} \mathrm{H}\right)^{-2}+\mathrm{O}\left(\mathrm{SNR}^{-3}\right) \\
& =\left(\mathrm{SNR} \cdot \mathrm{H}^{\mathrm{H}} \mathrm{H}\right)^{-1}\left[1-\mathrm{I}_{\mathrm{N}_{\mathrm{r}}}\left(\mathrm{SNR} \cdot \mathrm{H}^{\mathrm{H}} \mathrm{H}\right)^{-1}+\mathrm{O}\left(\mathrm{SNR}^{-2}\right)\right]
\end{aligned}
$$

Therefore, at high SNR condition, the capacity difference between LR-MMSE and MMSE can be expressed as,

$$
\begin{aligned}
& \mathrm{C}_{\mathrm{MMSE}}^{\mathrm{LR}}-\mathrm{C}_{\mathrm{MMSE}}=\log _{2}\left(\mathrm{SNR} \cdot \mathrm{H}^{\mathrm{H}} \mathrm{H}\right)-\log _{2}\left[1-\mathrm{I}_{\mathrm{N}}\left(\mathrm{SNR}_{\mathrm{H}} \mathrm{H}^{\mathrm{H}}\right)^{-1}+\mathrm{O}\left(\mathrm{SNR}^{-2}\right)\right]-\log _{2}\left(\mathrm{SNR} \cdot \widetilde{\mathrm{H}}^{\mathrm{H}} \widetilde{\mathrm{H}}\right) \\
& +\log _{2}\left[1-\mathrm{I}_{\mathrm{N}_{\mathrm{r}}}\left(\mathrm{SNR} . \widetilde{\mathrm{H}}^{\mathrm{H}} \widetilde{\mathrm{H}}\right)^{-1}+\mathrm{o}\left(\mathrm{SNR}^{-2}\right)\right] \\
& \mathrm{C}_{\mathrm{MMSE}}^{\mathrm{LR}}-\mathrm{C}_{\mathrm{MMSE}}=\log _{2}\left(\frac{\widetilde{\mathrm{H}}^{\mathrm{H}} \widetilde{\mathrm{H}}}{\mathrm{H}^{\mathrm{H}}}\right)+\log _{2}\left(\frac{1-\mathrm{I}_{\mathrm{N}_{\mathrm{r}}}\left(\mathrm{SNR} . \mathrm{H}^{H} H\right)^{-1}+\mathrm{o}\left(\mathrm{SNR}^{-2}\right)}{\left[1-\mathrm{I}_{\mathrm{N}_{\mathrm{r}}}\left(\mathrm{SNR} \cdot \widetilde{\mathrm{H}}^{\mathrm{H}} \widetilde{\mathrm{H}}\right)^{-1}+\mathrm{o}\left(\mathrm{SNR}^{-2}\right)\right]}\right)
\end{aligned}
$$

Equation (7) represent the capacity improvement due to LR-MMSE system.

\section{Simulation Results}

For the simulation purpose, authors have consider 4x4 MIMO system in correlated Nakagami-m and SCM channel environment. Here only the receiver side antenna correlation $(\rho)$ have been considered and also channel state information is available only at the receiver side. The overall simulation results are presented in two sections: performance in Nakagami-m channel followed by SCM channel.

\subsection{Nakagami-m Channel Model:}

This section represents the performance of the MIMO system in Nakagami-m channel environment. 


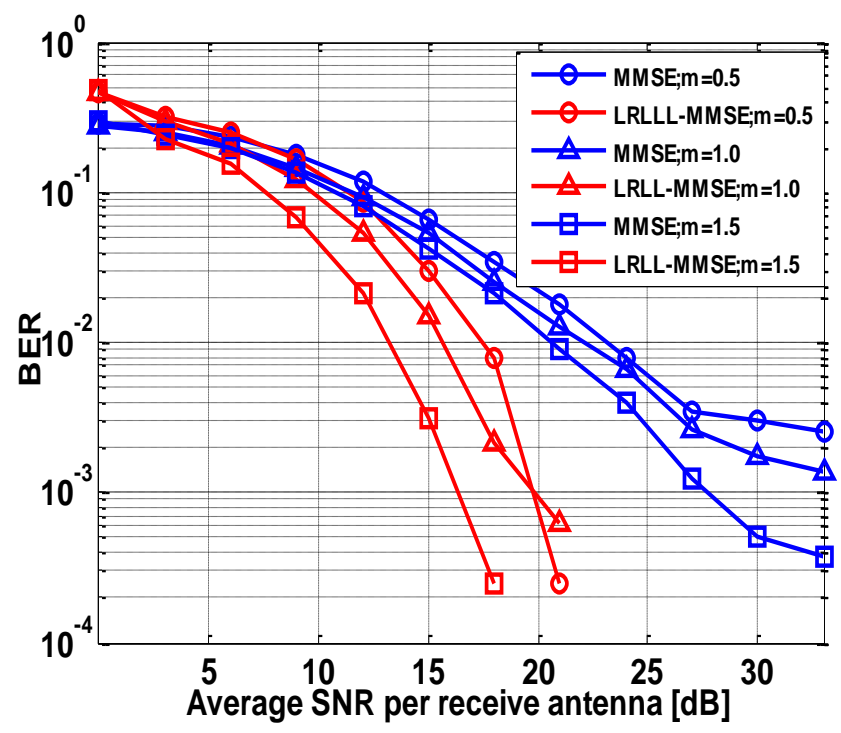

Fig.1. SNR Vs BER Curves for Different M.

As in figure 1, with increase in $\mathrm{m}$ value the BER performance of the system gets better. As represented in figure 3, for $m=1.5$, the required SNR to achieve a BER of $10^{-2}$ for all the systems are $13 \mathrm{~dB}$ (LRLLL-MMSE), $21 \mathrm{~dB}$ (MMSE), Therefore it clear that there is significant amount of SNR improvement in case of LRLLL aided system.

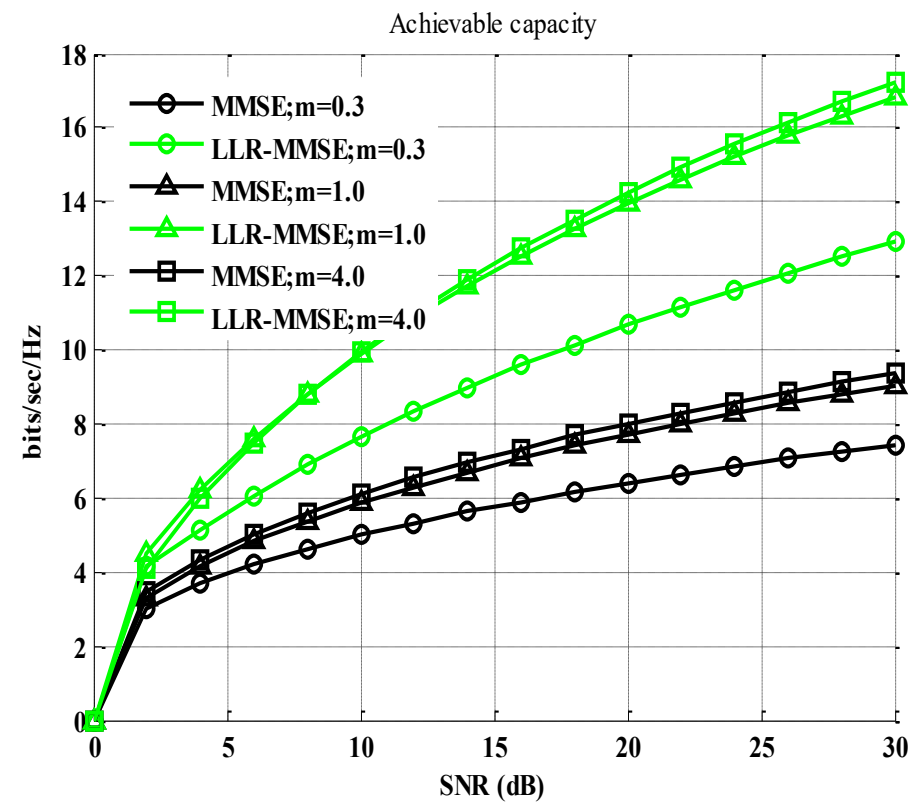

Fig.2. Channel Capacity with the Variation of $m$. 
Table 1. Channel Capacity Comparison for 4x4 MIMO System.

\begin{tabular}{|l|l|l|l|}
\hline \multirow{2}{*}{$\begin{array}{l}\text { Name of the } \\
\text { receiver }\end{array}$} & \multicolumn{3}{|l|}{ Channel Capacity (bps/Hz) at SNR of $26 \mathrm{~dB}$} \\
\cline { 2 - 4 } & $\mathrm{m}=0.3$ & $\mathrm{~m}=1.0$ & $\mathrm{~m}=4.0$ \\
\hline MMSE & 7.05 & 8.56 & 8.86 \\
\hline LLR-MMSE & 12.08 & 15.79 & 16.14 \\
\hline
\end{tabular}

As in Table 1, LLR-MMSE receiver bost the system performance effectively. Fig. 2 shows the comparetivee analysis of different receivers. It can also be seen that with the imcrease in SNR the performance gap between LLR-MMSE and MMSE increases. Let us consider the case for $\mathrm{m}=0.3$, the channel capacity at low SNR region (considering $\mathrm{SNR}=4 \mathrm{~dB}$ ) the capacities for MIMO system with LLR-MMSE and MMSE receivers are $5.153 \mathrm{bps} / \mathrm{Hz}$ and $3.67 \mathrm{bps} / \mathrm{Hz}$ respectively. Therefore, the capacity gap is about $1.483 \mathrm{bps} / \mathrm{Hz}$ in low SNR region. Now, in same channel condition at high SNR region (considering SNR=26dB) the capacities for MIMO system with LLR-MMSE and MMSE receivers are $12.08 \mathrm{bps} / \mathrm{Hz}$ and $7.055 \mathrm{bps} / \mathrm{Hz}$ respectively. Therefore, the capacity gap is about $5.025 \mathrm{bps} / \mathrm{Hz}$ in high SNR condition.

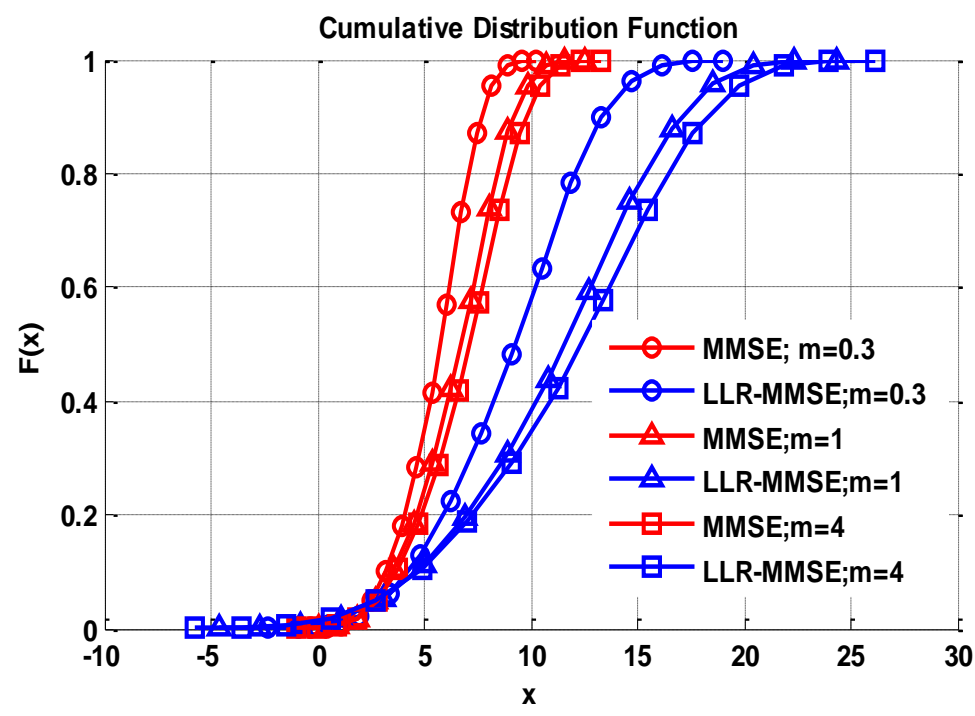

Fig.3. CDF of Channel Capacity with the Variation of $\mathrm{m}$.

From above Fig.3 3, it is clear that $m$ having significant impact on the system performance. As in Fig. LLRMMSE provide capacity gain in comparison to other receivers.

\subsection{With Spatial Channel Model (SCM):}

In this section performance of the MIMO system has been analyzed in SCM channel environment. 


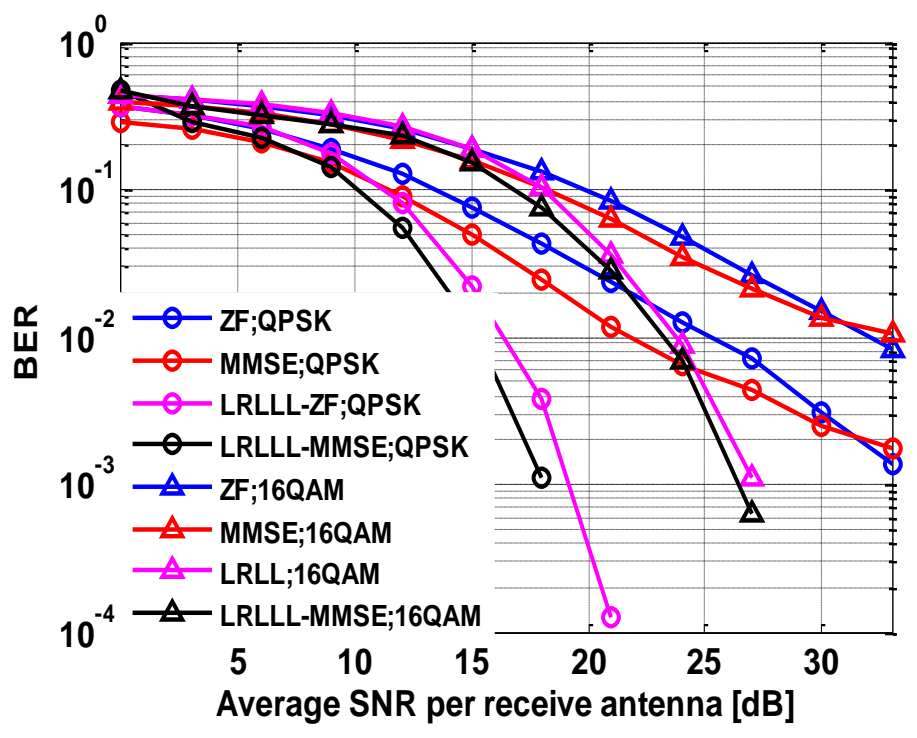

Fig.4. SNR Vs BER Curves for Different Modulations.

Fig. 4 represent the comparison of the performances of LRLLL concatenated ZF/MMSE receiver with conventional ZF and MMSE receiver with the variation modulation order. As in Fig. 4, for any modulation scheme ZF performs worst whereas LRLL-MMSE performs best. Let us consider QPSK modulation for performance evaluation. To achieve BER of the order of 10e-2, LRLLL-MMSE required $15 \mathrm{~dB}$ SNR whereas ZF needs SNR of 25dB. It shows by using LRLLL-MMSE receiver one can obtained a SNR gain of around $10 \mathrm{~dB}$.

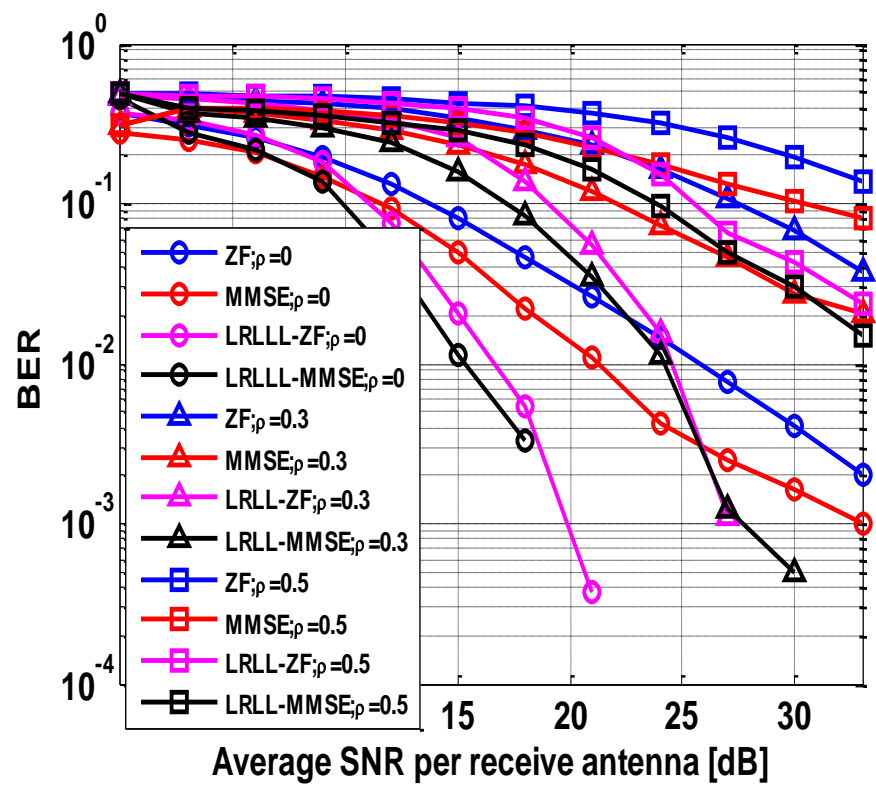

Fig.5. SNR Vs BER Curves for Different $\rho$. 
Fig. 5 depicts the impact of the channel correlation coefficient on the system performance. Its shows a significant improvement in system performance due to LRLLL-MMSE receiver.

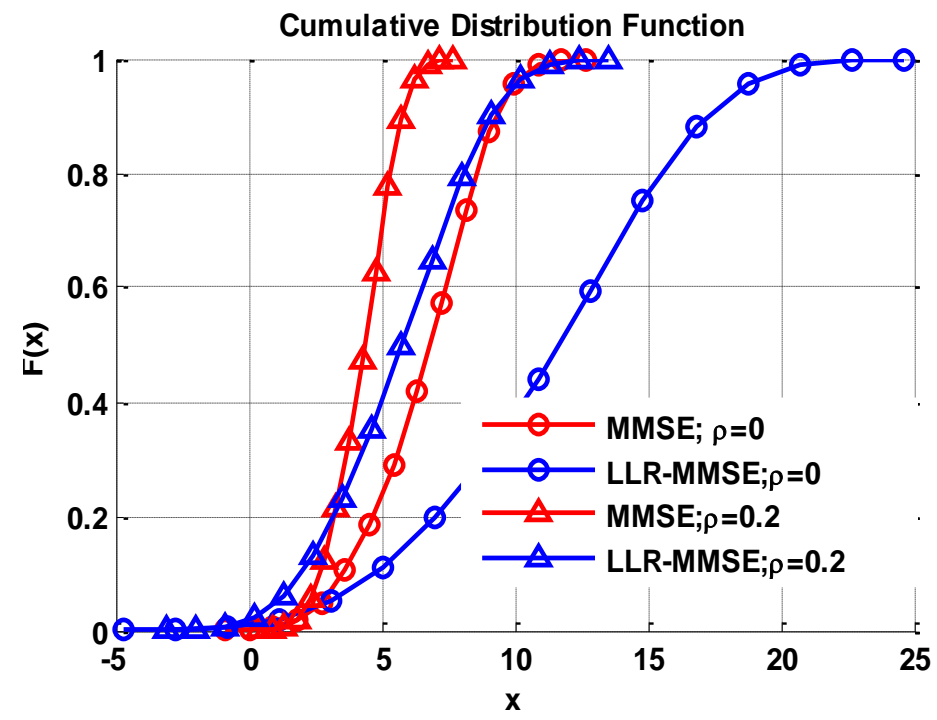

Fig.6. CDF of Channel Capacity with the Variation of $\rho$.

From above Fig. 6, it is clear that channel correlation coefficient having significant impact on the system performance. But LLR-MMSE performs much better than the conventional MMSE receivers. So, channel capacity improvement due to the CLLL aided receiver is prominent through the mathematical calculation and also the simulation results.

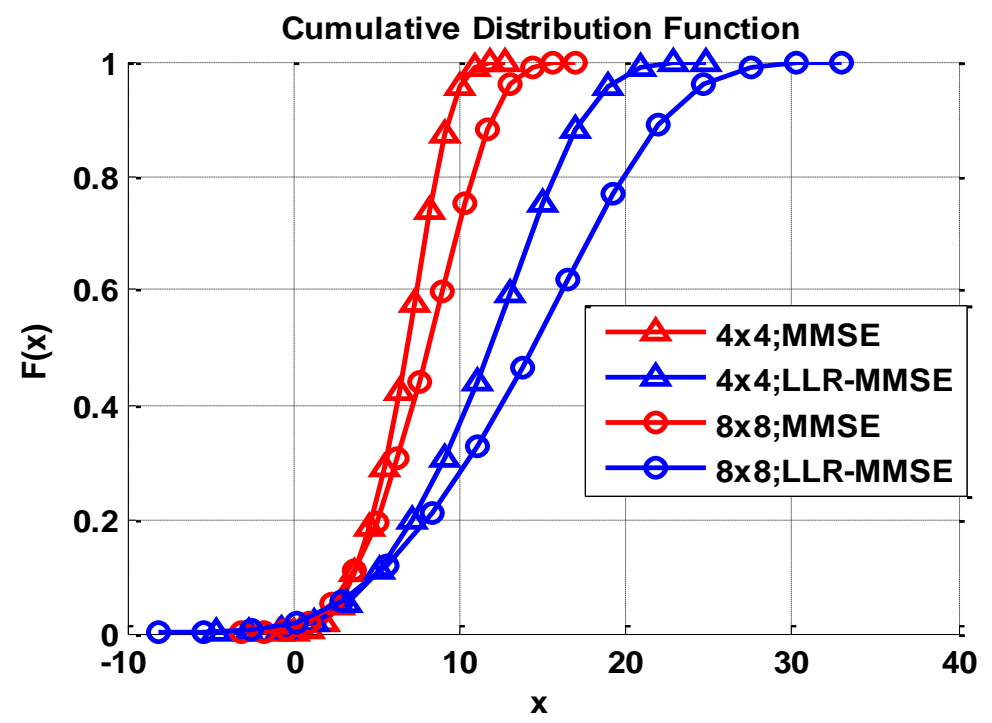

Fig.7. CDF of Channel Capacity with the Variation of Number of Antennas. 
As indicated in the Fig. 7, with the increase in the number of antennas have significantly increase the channel capacity. But the main observation of the above figure is that the rate of increase of the capacity is different for the MMSE and LLR-MMSE receivers. If we consider $\mathrm{F}(\mathrm{x})=0.9$ as reference level then the capacity gap for MMSE and LLR-MMSE receivers are 2.5 and $5 \mathrm{bps} / \mathrm{Hz}$. It clearly indicate that LLR-MMSE receiver provide low complex receiver with improved system performance.
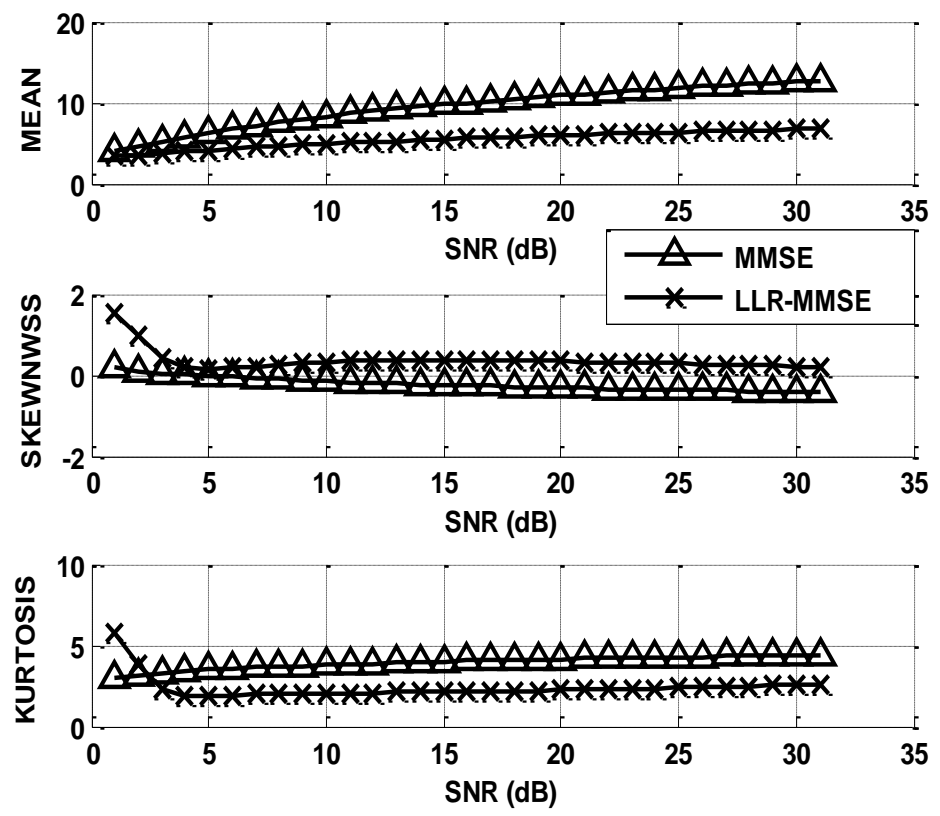

Fig.8. Mean, Skewness, and Kurtosis of the Capacity (bits/s/Hz) versus SNR for Nakagami-m (m=1) 2x2 MIMO Channels.

Fig. 8 shows the mean, variance, skewness, and kurtosis of MIMO channel capacity considering Nakagami$\mathrm{m}$ channel. This represent a comparative higher order statistical analysis of the MIMO channel capacity in presence of the linear receiver MMSE and nonlinear low complex receiver LLR-MMSE. From the above result, one can conclude that LLR-MMSE receiver provide more stable receiver system.

\section{Conclusions}

In this paper, performance of different receiver system have been analyzed in Nakagami-m and SCM channel condition. Different channel condition have been taken into account to validate the utility of LLR based receiver for the MIMO system.

$\mathrm{ZF}$ and MMSE are most commonly used receivers for MIMO system. But with the increase in number of antennas, these algorithm need to be modified to reduce the computation complexity. LLR based receiver architecture produces low complex systems. LLR aided receiver boost the system performance significantly. Through the mathematical model and the simulation result we have demonstrated the effectiveness of LLRMMSE receiver over the MMSE receiver. 


\section{References}

[1] Xiaoli M, Yiming K.: 'Capacity Analysis of Lattice Reduction Aided Equalizers for MIMO Systems', IEEE MILCOM-2015, December 2015, pp.866-871.

[2] C.-J. Chen and L.-C. Wang, 'On the performance of the zero-forcing receiver operating in the multiuser MIMO system with reduced noise enhancement effect,', IEEE Global Communications Conf. (GLOBECOM), St. Louis, MO, Nov 2005, pp. 1294-1298.

[3] X. Ma and W. Zhang, "Fundamental limits of linear equalizers: Diversity, capacity, and complexity," IEEE Trans. Inf. Theory, Aug. 2008, 54, (8), pp. 3442-3456.

[4] Yao. H. and Wornell. G. W., 'Lattice-reduction-aided detectors for MIMO communication systems,' IEEE Global Tele. Conf., Taipei,Taiwan, Nov. 2002, pp. 424-428.

[5] Berenguer. I, Adeane. J, Wassell. I.J., and Wang. X, "Lattice-reductionaided receiver for MIMO-OFDM in spatial multiplexing systems," Proc.IEEE GLOBECOM, Nov. (2005).

[6] Y. H. Gan and H. W. Mow, "Complex lattice reduction algorithm for lowcomplexity MIMO detection," Proc. IEEE PIMRC, Vol. 2, pp. 1516-1521, (Sep. 2004).

[7] D. W“ ubben, D. Seethaler, J. Jald' en, and G. Matz, "Lattice reduction," IEEE Signal Process. Mag., vol. 28, no. 3, pp. 70-91, (May 2011).

[8] J. Jalden and P. Elia, "DMT optimality of LR-aided linear decoders for a general class of channels, lattice designs, and system models," IEEE Trans. Inform. Theory, vol. 56, no. 10, pp. 4765-4780, (Oct. 2010).

[9] A. K. Singh, P. Elia, and J. Jalden, "Achieving a vanishing SNR gap to exact lattice decoding at a subexponential complexity,” IEEE Trans. Inform. Theory, vol. 58, no. 6, pp. 3692-3707, (Jun. 2012).

[10] Chun-Fu Liao, Li-Wei Chai, and Yuan-Hao Huang, "Loop-Reduction LLL Algorithm and Architecture for Lattice-Reduction-Aided MIMO Detection", Journal of Electrical and Computer Engineering Volume 2012, pp-1-8, (2012).

[11] Y. H. Gan, C. Ling, and W. H. Mow, "Complex lattice reduction algorithm for low-complexity fulldiversity MIMO detection," IEEE Transactions on Signal Processing, vol. 57, no. 7, pp. 2701-2710, (2009).

[12] Samarendra Nath Sur, Soumyasree Bera, Arun Kumar Singh, Rabindranath Bera and Bansibadan Maji, "Performance Analysis of VBLAST Based MIMO OFDM System in Vehicular Channel", International Journal of Intelligent Systems and Applications(IJISA), Vol-6, N0. 12, November, 2014, pp- 62-68.

[13] Samarendra Nath Sur, Debjyoti Ghosh, "Channel Capacity and BER Performance Analysis of MIMO System with Linear Receiver in Nakagami Channel", I.J.Wireless and Microwave Technologies, 2013, Vol.1, pp-26-36.

[14] Samarendra Nath Sur and Rabindranath Bera, "Doppler Shift Impact on the MIMO OFDM System In Vehicular Channel Condition”, I.J. Information Technology and Computer Science (IJITCS), Vol. 4, No. 8, 2012, pp 57-68.

[15] Sur S. N, Bera. S, Bera. R. and B. Maji, 'MIMO Channel Capacity in Non-Uniform Phase Distributed Nakagami Channel with ZF Receiver', 3rd International Conference on Foundations and Frontiers in Computer, Communication and Electrical Engineering (C2E2 - 2016), 15th - 16th January 2016, pp. 289-293, doi. 10.1201/b20012-58. 


\section{Authors' Profiles}

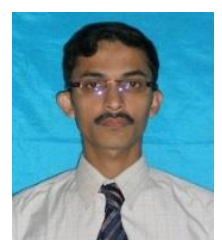

Samarendra Nath Sur: Born in Hooghly, West Bengal, India, in 1984. He received B.Sc degree in Physics (Hons.) from the University of Burdwan in 2007. He received M.Sc. degree in Electronics Science from Jadavpur University in 2007 and M.Tech degree in Digital Electronics and Advanced Communication from Sikkim Manipal University in 2012. $\mathrm{He}$ is currently working towards the Ph.D degree in Electronics and Communication Engineering at NIT, Durgapur.

Since 2008, he has been associated with the Sikkim Manipal Institute of Technology, India, where he is currently an assistant professor in the department of Electronics \& Communication Engineering. His current research interests include Broadband Wireless Communication (MIMO and Spread Spectrum Technology), Advanced Digital Signal Processing and Remote Sensing. He is a Member of Institute of Electrical and Electronics Engineers (IEEE) and International Association of Engineers (IAENG).

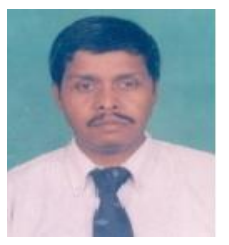

Rabindranath Bera: He is a Professor and Ex- Dean (R\&D) in Sikkim Manipal University and Ex- Reader of Calcutta University, West Bengal, India. B.Tech, M.Tech and Ph.D from Radio-Physic \& Electronics, Calcutta University. His Research areas are Digital Radar, RCS Imaging, 4G Communication, Radiometric remote sensing. He has published a large number of papers in different national \& international Conferences and Journals.

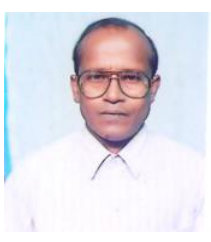

Bansibadan Maji: $\mathrm{He}$ is currently working as a senior Professor of Electronics \& Communication Engineering Department in NIT, Durgapur, West Bengal, India. He is former Head of the Department of ECE at NIT. His main research area on Microwave, Antenna, VLSI Design and Low power Device and Circuits. He has more than 56 publications in different International and National Journals and Conference Proceedings

How to cite this paper: Samarendra Nath Sur, Rabindranath Bera, Bansibadan Maji," Lattice-ReductionAided Equalization for V2V Communication Channel", International Journal of Wireless and Microwave Technologies(IJWMT), Vol.8, No.2, pp. 64-73, 2018.DOI: 10.5815/ijwmt.2018.02.06 